\title{
Serum hexosaminidase and ß-glucuronidase activities in infants: effects of age and sex
}

\section{P. Mabe ${ }^{1,2}$ and M. Beck ${ }^{2}$}

\section{Correspondence \\ P. Mabe \\ Unidad de Genética y Enfermedades Metabólicas \\ INTA, Universidad de Chile \\ Casilla 138-1 \\ Santiago de Chile \\ Chile \\ Fax: + 56-2-294-1254 \\ E-mail: pmabe@uec.inta.uchile.cl}

Part of the Doctoral thesis presented by $\mathrm{P}$. Mabe to the JohannesGutenberg Universität, Maina, Germany. P. Mabe was supported by the Dr. Kottmeier Foundation

Received April 2, 2002 Accepted November 12, 2002
${ }^{1}$ Unidad de Genética y Enfermedades Metabólicas, INTA, Universidad de Chile, Santiago, Chile

${ }^{2}$ Biochemisches Labor-Kinderklinik der Universität Mainz, Mainz, Germany

\begin{abstract}
We investigated the effect of age and sex on the serum activity of hexosaminidase (HEX) and B-glucuronidase (BGLU) in 275 normal term infants aged $12 \mathrm{~h}$ to 12 months. Up to six weeks of life, HEX was significantly higher in boys $(\mathrm{P} \leq 0.023)$. During the age period of $1-26$ weeks, BGLU was also higher in boys, but differences were significant only at 2-6 and 7-15 weeks $(\mathrm{P} \leq 0.016)$. The developmental pattern of HEX and BGLU was sex dependent. HEX activity increased in both sexes from 4-7 days of life, reaching a maximum of 1.4-fold the birth value at 2-6 weeks of age in boys $(\mathrm{P}<0.001)$ and a maximum of 1.6fold at $7-15$ weeks in girls $(\mathrm{P}<0.001)$. HEX activity gradually decreased thereafter, reaching significantly lower levels at 27-53 weeks than during the first three days of life in boys $(\mathrm{P}=0.002)$ and the same level of this age interval in girls. BGLU increased in both sexes from 4-7 days of age, showing a maximum increase at 7-15 weeks (3.3-fold in boys and 2.9-fold in girls, both $\mathrm{P}<0.001$ ). Then BGLU decreased in boys to a value similar to that observed at 4-7 days of age. In girls, BGLU remained elevated until the end of the first year of life. These results indicate a variation of HEX and BGLU activities during the first year of life and a sex influence on their developmental pattern. This observation should be considered in the diagnosis of $\mathrm{GM}_{2}$ gangliosidosis and mucopolysaccharidosis type VII.
\end{abstract}

\section{Introduction}

Congenital deficiency of the lysosomal acid hydrolases $\mathrm{N}$-acetyl- $\beta$-hexosaminidase $\mathrm{A}$ and $\mathrm{B}$ (HEX, EC 3.2.1.30) or of ß-glucuronidase (BGLU, EC 3.2.1.31) is the cause of the autosomal recessive inherited disorders $\mathrm{GM}_{2}$ gangliosidosis and mucopolysaccharidosis type VII (McKusick 253220), respectively.

$\mathrm{GM}_{2}$ gangliosidosis includes three different biochemical patterns: i) Tay-Sachs disease or variant B (McKusick 272800), sec-
Key words

- Lysosomal storage disease

- $\mathrm{GM}_{2}$ gangliosidosis

- Mucopolysaccharidosis

type VII

- Hexosaminidase

- ß-Glucuronidase ondary to deficiency of the isoenzyme HEX A, ii) Sandhoff disease or variant 0 (McKusick 268800) caused by deficiency of the isoenzymes HEX A and HEX B, and iii) variant AB (McKusick 272750) secondary to $\mathrm{GM}_{2}$ activator deficiency. The biochemical diagnosis of $\mathrm{GM}_{2}$ gangliosidosis and mucopolysaccharidosis type VII, whose clinical and biochemical characteristics are well described $(1,2)$, is usually established by measuring the HEX and BGLU activities in serum using 4-methyl-umbelliferyl derivatives as substrates. At present, no treatment 
is available for these neurodegenerative diseases. Current research efforts are focused on the introduction of a functional enzyme through allogeneic bone marrow transplantation, replacement with purified or recombinant enzyme or gene therapy (3-6), and also substrate deprivation (7).

Because the genetic trait for Tay-Sachs disease is highly frequent among Jewish Ashkenazi groups (heterozygote frequency, 1:28) (8) and in French Canadian populations (heterozygote frequency, 1:66) (9), a screening program for the detection of heterozygotic subjects has been successfully implemented (10). This strategy has drastically decreased the incidence of this severe neurodegenerative disorder.

Since heterozygotes for $\mathrm{GM}_{2}$ gangliosidosis and mucopolysaccharidosis type VII achieve HEX or BGLU levels close to $50 \%$ the normal values, respectively, they could potentially be detected by measurement of these enzymes in leukocytes or serum. In infants, however, the changes of HEX A, HEX B and BGLU activities in serum along the first year of life are unclear, impairing the detection of heterozygotes for $\mathrm{GM}_{2}$ gangliosidosis and mucopolysaccharidosis type VII during this age period.

The objective of the present study was to characterize the developmental pattern of total serum HEX (HEX AB), HEX A, HEX $\mathrm{B}$ and BGLU activities during the first year of life, taking into account the influence of both age and gender. A better understanding of the behavior of these lysosomal enzymes should improve the diagnosis of the $\mathrm{GM}_{2}$ gangliosidosis and mucopolysaccharidosis type VII conditions, especially for the heterozygote condition.

\section{Material and Methods}

\section{Subjects}

The protocol was explained to parents whose infants were admitted to the newborn nursery or for minor elective surgery in the Pediatric Unit, University Clinical Hospital, Mainz, Germany. Informed consent was obtained in 275 cases, all of whom were apparently healthy German children, except for the minor surgical pathology that motivated admission (tonsillectomy, adenotomy, circumcision, and uncomplicated inguinal and umbilical hernia repair). They were feeding and growing normally and were receiving no pharmacological treatments. Ages ranged from $12 \mathrm{~h}$ to 12 months and gestational ages from 38 to 42 weeks. The children (136 boys and 139 girls) were divided into six age categories: 1) 0-3 days, 2) 4-7 days, 3) 2-6 weeks, 4) 7-15 weeks, 5) 16-26 weeks and 6) 27-53 weeks on the basis of ANOVA of the results, which showed no differences according to gestational age and suggested this age categorization to optimize differences between groups.

\section{Samples}

During routine blood sampling, an additional $0.5 \mathrm{ml}$ of blood was obtained without anticoagulant. The samples were centrifuged and serum was stored at $-20^{\circ} \mathrm{C}$ until assayed within two weeks. Because an earlier study on newborn infants had revealed fluctuation in serum HEX activity according to a circadian cycle (11), samples were always collected between 8:00 and 10:00 h.

\section{Biochemical measurements}

The activities of isoenzymes HEX A, HEX B and of BGLU were determined fluorimetrically using analytical grade 4-methylumbelliferyl derivatives (Sigma, St. Louis, MO, USA). HEX activities were assayed after heat inactivation (12). Briefly, HEX $A B$ activity was first determined, then heat-labile HEX A was inactivated and the new measurement yielded HEX B activity. HEX A activity was calculated as the difference between total HEX AB and HEX B. 
The BGLU assay was performed using the fluorometric method described by Woollen and Walker (13). Enzyme activity is reported as $\mathrm{mU} / \mathrm{ml}$ serum; one milliunit ( $\mathrm{mU}$ ) of HEX and BGLU activity is defined as $1 \mathrm{nmol}$ of 4-methyl-umbelliferone released per minute.

\section{Statistical analysis}

For statistical analysis of the data, means and standard deviations of total HEX AB, HEX A, HEX B and BGLU activities were calculated and tested for statistical significance by ANOVA for comparison between age groups and by the Student $t$-test for comparison between genders, with the level of significance set at $\mathrm{P} \leq 0.05$.

\section{Results}

The data in Figure 1 indicate that the HEX AB activity increased gradually from the age interval of 4-7 days to a 1.4-fold maximum at the age interval of 2-6 weeks in boys (ANOVA, $\mathrm{P}<0.001$ ) and to a 1.6 -fold maximum at 7-15 weeks in girls (ANOVA, $\mathrm{P}<0.001)$. HEX A and HEX $\mathrm{B}$ levels also rose in both sexes from 4-7 days, reaching a maximal increase at the same age interval as HEX AB. However, the elevation of HEX A was higher than that of HEX B. HEX A reached a maximal increase of 1.6-fold in boys and of 1.7-fold in girls (ANOVA for both, $\mathrm{P}<0.001$ ); the HEX $\mathrm{B}$ increase was 1.3-fold in boys (ANOVA, $\mathrm{P}=0.003$ ) and 1.4-fold in girls (ANOVA, $\mathrm{P}<0.001$ ). Subsequently, HEX AB and HEX B decreased until the age period of 27-53 weeks, reaching a significantly lower level (ANOVA, $\mathrm{P}=$ 0.002 and $\mathrm{P}<0.001$ for HEX AB and HEX B, respectively) than in the first three days of life in boys and the same level as at 0-3 days in girls. HEX A declined in parallel to HEX $\mathrm{AB}$ and HEX $\mathrm{B}$, reaching the same values at the age interval of 27-53 weeks as in the first three days of life in boys and as the 4-7 days of life in girls. The sharpest decline was in
HEX B activity, reaching 2.3-fold in boys and 1.6-fold in girls. HEX A reduction was 1.7-fold in boys and 1.4-fold in girls, while HEX AB decreased 1.9-fold in boys and 1.5fold in girls. All HEX reductions were highly significant (ANOVA, $\mathrm{P}<0.001$ ).

During the first six weeks of life HEX $A B$ and HEX $B$ values were significantly higher in boys than in girls ( $t$-test, $\mathrm{P} \leq 0.023$ ). While HEX A also tended to be greater in boys, differences were significant only during the age interval of 2-6 weeks ( $t$-test, $\mathrm{P}=$ 0.012 ). At 7-15, 16-26 and 27-53 weeks of life, the serum HEX AB, HEX A and HEX B activities tended to be slightly higher in girls than in boys, but the differences were not statistically significant.

Serum BGLU activity also increased from 4-7 days of age to a maximal rise of 3.3-fold during the 7-15-week interval in boys and of 2.9-fold in girls (ANOVA for both, $\mathrm{P}<0.001$ ). Then, BGLU values decreased 1.4-fold in boys (ANOVA, $\mathrm{P}=0.001$ ) reaching slightly but not significantly higher levels during the 27-53-week period compared to the 4-7-day period. In girls, however, serum BGLU levels continued to be elevated until the end of the first year of life. When BGLU activities were compared according to sex, the level tended to be higher in boys than in girls until the age period of 16-26 weeks, with a significant difference during the age intervals of 26 weeks $(t$-test, $\mathrm{P}<0.001)$ and $7-15$ weeks $(t$ test, $\mathrm{P}=0.016)$. In the age group of 27-53 weeks, BGLU values tended to be higher in girls than in boys, but the difference was not significant.

\section{Discussion}

The aim of this study was to characterize the developmental pattern of the serum HEX and BGLU enzyme activities during the first 12 months of life. Results for apparently healthy children revealed variations in both serum HEX and BGLU activities during the first year of life and differences between 


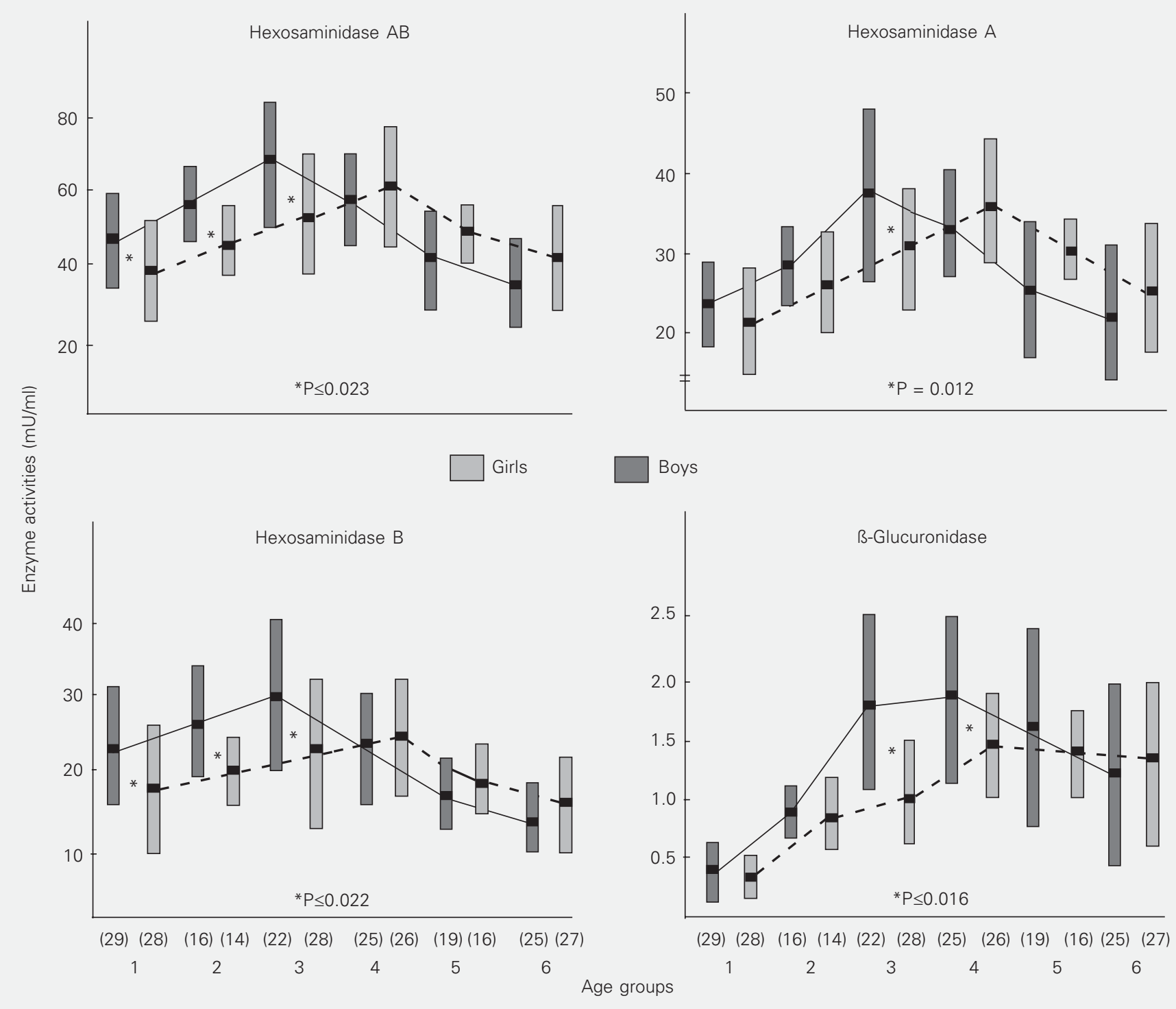

ANOVA also revealed significant differences $(P \leq 0.037)$ between the following age groups:

Boys

$\begin{array}{lllll}\text { HEX AB: } & 1 \text { and } 2,3,4,6 & 2 \text { and } 3,5,6 & 3 \text { and } 4,5,6 & 4 \text { and } 5,6 \\ \text { HEX A: } & 1 \text { and } 3,4 & 2 \text { and } 3,4,6 & 3 \text { and } 5,6 & 4 \text { and } 5,6 \\ \text { HEX B: } & 1 \text { and 3,5,6 } & 2 \text { and 5,6 } & 3 \text { and } 4,5,6 & 4 \text { and } 5,6 \\ \text { BGLU: } & 1 \text { and 2,3,4,5,6 } & 2 \text { and 3,4,5 } & 3 \text { and } 6 & 4 \text { and } 6 \\ & & & & \\ \text { HEX AB: } & 1 \text { and 3,4,5 } & 2 \text { and } 4 & 3 \text { and } 4,6 & 4 \text { and } 5,6 \\ \text { HEX A: } & 1 \text { and 3,4,5,6 } & 2 \text { and } 4 & 3 \text { and } 4,6 & 4 \text { and } 5,6 \\ \text { HEX B: } & 1 \text { and 3,4 } & & 3 \text { and } 6 & 4 \text { and } 5,6 \\ \text { BGLU: } & 1 \text { and } 2,3,4,5,6 & 2 \text { and 4,5,6 } & 3 \text { and } 4,5,6 & \end{array}$

Figure 1. Total serum hexosaminidase (HEX AB), hexosaminidase A (HEX A), hexosaminidase $B$ (HEX B) and B-glucuronidase (BGLU) activities according to age period and sex. Results are reported as mean $\pm \mathrm{SD}$ enzyme activities in $\mathrm{mU} / \mathrm{ml}$. Numbers in parentheses below the Figure indicate the number of subjects per group. The age groups of 0-3 days, 4-7 days, 2-6 weeks, 7-15 weeks, 16-26 weeks, and 27-53 weeks are represented by the numbers 1, 2, 3, 4, 5 and 6, respectively. Enzyme activities were compared between girls and boys by the Student $t$-test and between the different age groups according to sex by two-way ANOVA. 
sexes. Previous reports have suggested an influence of age on HEX and BGLU activities, showing higher levels of serum HEX $(12,14)$ and BGLU activities (13) during the first years of life compared to those of prepuberal children and adults. Reports by Tenore et al. (15), Oberkotter et al. (16) and Shattuck et al. (17) suggested that serum HEX activity changes along the first six months of life while serum BGLU activity varies during the first 12 months (18). These studies, however, did not evaluate the second six months of life or did not include sufficient individuals and thus did not provide an analysis of the developmental pattern along the entire first year of life. The conclusions of the earlier investigations related to the influence of sex on serum HEX activity in infants are divergent (15-17). Likewise, the influence of sex on serum BGLU activity during the first year of life has not been systematically investigated previously (18).

Our results demonstrated a gradual increase of HEX starting during the first days of life to a maximal rise during the first three months. Subsequently, the enzyme activity decreased, reaching in the second semester the same or significantly lower levels than those observed in the first three days of life. These results agree with previous data reported on the developmental pattern of serum HEX activity during the first semester of life (15-17) and add information about the changes of its levels during the second semester.

The factors that could explain the higher serum HEX and BGLU activities in infants than in older children and adults and the variability of these enzyme levels during the first year of life are unknown. In an attempt to elucidate this last aspect, Tenore et al. (15) found a positive correlation between the changes of serum HEX and thyroxin levels in newborns. The potential role of thyroxin in HEX activity has been formerly suggested by the finding of approximately $50 \%$ of the normal HEX level in skeletal muscle and liver of thyroidectomized rats (19). A positive correlation between thyroxin level and serum HEX activity has been reported in euthyroid, hypothyroid and hyperthyroid prepuberal females (20), suggesting the influence of this hormone on human HEX activity as well. Because newborns show a similar pattern of increase and subsequent decrease for both proteins, the higher serum HEX activity of infants in comparison to older children could be explained, at least in part, through its regulation by this hormone. Further support for this hypothesis is provided by studies on adults, in whom a positive correlation between these two parameters was also observed (21).

Another possible explanation for high lysosomal enzyme activities in the serum of infants could be the secretion of these proteins from organs that are especially active during this period of life. Data from an earlier study suggested that one of these organs could be the thymus (22). Both HEX and BGLU activities have been measured in a variety of cadaver organs and the highest levels were observed in infant thymus. The values obtained were considerably higher for both enzymes, even higher than those measured in adult livers.

Oberkotter et al. (16) proposed that the higher serum HEX activity of male infants might also be due to hormonal factors. These authors have observed a high degree of correlation between testosterone concentration and serum HEX activity in both sexes, suggesting a regulatory role of the male hormone.

According to data obtained in a murine model (23), the BGLU activity of a male muscle homogenate was higher than that of females. This difference tended to disappear after orchidectomy and reappeared when the orchidectomized mice received testosterone. These results suggest that BGLU levels could also be influenced by the male hormone. A similar conclusion was reached by Molano et al. (24) in a study in which higher BGLU 
levels were observed in liver homogenates from androgenic steroid-treated rats than in liver homogenates from untreated animals.

However, although common hormonal factors are likely to play a role in the regulation of HEX and BGLU activities, it is also possible that other undetermined factors are relevant. These could explain the higher incremental changes in BGLU compared with HEX activity and the different developmental pattern of these enzymes in girls during the first year of life.

The results of this study show a considerable variation of serum HEX and BGLU activities during the whole first year of life and sex dimorphism in their developmental pattern. We conclude that the influence of age and gender should be considered when $\mathrm{GM}_{2}$ gangliosidosis and mucopolysaccharidosis type VII (or their heterozygotic conditions) are tested by serum HEX or BGLU analysis in infants.

\section{Acknowledgments}

The authors are grateful to Dr. M. Araya and Dr. I. Atwater for helpful discussion and manuscript revision, and to Ms. R. Hellmann and Ms. V. Lyon for excellent technical assistance.

\section{References}

1. Neufeld E \& Muenzer J (2001). The mucopolysaccharidoses. In: Scriver Ch, Beaudet A, Sly W \& Valle D (Editors), The Metabolic and Molecular Bases of Inherited Disease. McGraw-Hill, Inc., New York, NY, USA.

2. Gravel R, Kaback M, Proia R, Sandhoff K, Suzuki K \& Suzuki K (2001). The GM2 gangliosidoses. In: Scriver Ch, Beaudet A, Sly W \& Valle D (Editors), The Metabolic and Molecular Bases of Inherited Disease. McGraw-Hill, Inc., New York, NY, USA.

3. Norflus F, Tifft CJ, McDonald MP, Goldstein G, Crawley JN, Hoffmann A, Sandhoff K, Suzuki K \& Proia RL (1998). Bone marrow transplantation prolongs life span and ameliorates neurologic manifestations in Sandhoff disease mice. Journal of Clinical Investigation, 101: 1881-1888.

4. Wada R, Tifft CJ \& Proia RL (2000). Microglial activation precedes acute neurodegeneration in Sandhoff disease and is suppressed by bone marrow transplantation. Proceedings of the National Academy of Sciences, USA, 97: 10954-10959.

5. Guidotti JE, Mignon A, Haase G, Caillaud C, McDonell N, Kahn A \& Poenaru $L$ (1999). Adenoviral gene therapy of the Tay-Sachs disease in hexosaminidase A-deficient knock-out mice. Human Molecular Genetics, 8: 831-838

6. Vogler C, Sands MS, Galvin N, Levy B, Thorpe C, Barker J \& Sly W (1998). Murine mucopolysaccharidosis type VII: the impact of therapies on the clinical course and pathology in a murine model of Iysosomal storage disease. Journal of Inherited Metabolic Disease, 21: $575-586$.

7. Jeyakumar M, Norflus F, Tifft CJ, Cortina-Borja M, Butters TD, Proia RL, Perry VH, Dwek RA \& Platt FM (2001). Enhanced survival in Sandhoff disease mice receiving a combination of substrate deprivation therapy and bone marrow transplantation. Blood, 97: 327-329.

8. Mitchell JJ, Capua A, Clow C \& Scriver CR (1996). Twenty-year outcome analysis of genetic screening programs for Tay-Sachs and beta-thalassemia disease carriers in high schools. American Journal of Human Genetics, 59: 793-798.

9. Prence EM, Jerome CA, Triggs-Raine BL \& Natowicz MR (1997). Heterozygosity for Tay-Sachs and Sandhoff diseases among Massachusetts residents with French Canadian background. Journal of
Medical Screening, 4: 133-136.

10. Kaback M, Lim-Steele J, Dabholkar D, Brown D, Levy N \& Zeiger K (1993). Tay-Sachs disease-carrier screening, prenatal diagnosis, and the molecular era. An international perspective, 1970 to 1993. The International TSD Data Collection Network. Journal of the American Medical Association, 270: 2307-2315.

11. Wilf-Miron R, Peleg L, Goldman B \& Ashkenazi IE (1992). Rhythms of enzymatic activity in maternal and umbilical cord blood. Experientia, 48: 520-523.

12. O'Brien JS, Okada S, Chen A \& Fillerup D (1970). Detection of heterozygotes and homozygotes by serum hexosaminidase assay. New England Journal of Medicine, 283: 15-20.

13. Woollen JW \& Walker PG (1965). The fluorometric estimation of ßglucuronidase in blood plasma. Clinica Chimica Acta, 12: 659-670.

14. Lombardo A, Goi GC, Marchesini S, Caimi L, Moro M \& Tettamanti G (1981). Influence of age and sex on five human plasma lysosomal enzymes assayed by automated procedures. Clinica Chimica Acta, 113: 141-152.

15. Tenore A, Oberkotter LV \& Koldovsky O (1980). Age- and sexassociated differences in the relationship of serum hexosaminidase and T4 levels in human neonates. Early Human Development, 4: 4149.

16. Oberkotter L, Tenore A \& Koldovsky O (1980). Sex differences in serum levels of $\mathrm{N}$-acetyl-ß-hexosaminidase in infancy: correlation of enzyme activity with testosterone levels. Early Human Development, 4: 325-332.

17. Shattuck K, Richardson CJ, Rassin D \& Lobe Th (1986). Development of serum hexosaminidase activity in infants. Biology of the Neonate, 49: 126-131.

18. Heringova A, Koldovsky $O$ \& Jirsova $V$ (1966). ß-glucuronidase activity in the serum of infants. Acta Paediatrica Scandinavica, 55: 398400.

19. DeMartino GN \& Goldberg AL (1978). Thyroid hormones control Iysosomal enzyme activities in liver and skeletal muscle. Proceedings of the National Academy of Sciences, USA, 75: 1369-1373.

20. Oberkotter LV, Tenore A, Palmieri MJ \& Koldovsky O (1979). Relationship of Exp thyroid status and serum N-acetyl-beta-glucosamini- 
dase isoenzyme activities in humans. Clinica Chimica Acta, 94: 281286.

21. Weissmann GO \& Segal RL (1970). Serum beta-glucuronidase in thyroid disease. Proceedings of the Society for Experimental Biology and Medicine, 134: 812-813.

22. Platt D \& Hartmann R (1967). Hyaluronidase, ß-Glucuronidase- und ß-Acetylglucosaminidase-Aktivität in epithelialen und mesenchymalen menschlichen Geweben. Klinische Wochenschrift, 45: 998-
1004

23. Koenig H, Goldstone A \& Lu CY (1980). Androgens regulate mitochondrial cytochrome c oxidase and lysosomal hydrolases in mouse skeletal muscle. Biochemical Journal, 192: 349-353.

24. Molano F, Saborido A, Delgado J, Morán M \& Megías A (1999). Rat liver lysosomal and mitochondrial activities are modified by anabolicandrogenic steroids. Medicine and Science in Sports and Exercise, 31: 243-250. 